\title{
Sensory Profile of Italian Craft Beers, Beer Taster Expert versus Sensory Methods: A Comparative Study
}

\author{
Chiara Medoro*, Marta Cianciabella, Francesca Camilli, Massimiliano Magli, Edoardo Gatti, \\ Stefano Predieri \\ INSTITUT OF BIOMETEOROLOGY-BIOAGRIFOOD DEPARTMENT, CNR, Bologna, Italy \\ Email: *c.medoro@ibimet.cnr.it
}

Received 28 April 2016; accepted 27 May 2016; published 30 May 2016

Copyright (C) 2016 by authors and Scientific Research Publishing Inc.

This work is licensed under the Creative Commons Attribution International License (CC BY).

http://creativecommons.org/licenses/by/4.0/

(c) (i) Open Access

\begin{abstract}
In recent years, the interest on craft beer has been increasingly growing. In this work, sensory traits of five Italian artisanal beers were explored by a trained panel, through different sensory analysis methods: Quantitative Descriptive Analysis (QDA) and a sensory dynamic method, Temporal Dominance of Sensations (TDS). The sensory profiles obtained through these methods were compared to the description given by an expert beer taster. The trained panel $(n=12)$ evaluated five Tuscan beers, manufactured in Maremma area, Tuscany region, first through QDA. Twentyeight sensory properties (visual, tactile, flavor and aromatic traits) were evaluated through a nine point scale. The descriptive profile was enriched by a dynamic sensory evaluation method, TDS. TDS was used by panelists to obtain a "real-time" flavor profile of the craft beers. During tasting, TDS provided information on the most striking flavor traits chosen among: floral, honey, roasted, chestnut, spicy, fruity, hoppy, and malty, of each beer. A PCA analysis showed the importance of the flavor attributes for beer profile compared to the expert taster description. Results highlighted the main traits of each beer and showed the validity of different profile methods. The interesting outcomes both provided useful profile patterns for brewers aiming at targeting specific segments of beer market and supported the development of interesting instruments for beer sensory analysis.
\end{abstract}

\section{Keywords}

Sensory Analysis, TDS, Craft Beers

\section{Introduction}

Beer is the most widely consumed alcoholic beverage in the world [1], its production in Italy accounts for 13.5 ${ }^{*}$ Corresponding author.

How to cite this paper: Medoro, C., Cianciabella, M., Camilli, F., Magli, M., Gatti, E. and Predieri, S. (2016) Sensory Profile of Italian Craft Beers, Beer Taster Expert versus Sensory Methods: A Comparative Study. Food and Nutrition Sciences, 7, 454465. http://dx.doi.org/10.4236/fns.2016.76047 
million hectoliters per year, representing a key player in the European beer market [2]. Lately, together with multinational companies, independent craft breweries have been flourishing, even in countries where there is not a strong beer making tradition, such as in Italy [3]. Italian beer consumers are increasing more and more and beer lovers are actually comparable to wine lovers [2]. Typical features of Italian craft breweries are the care for enhancing the value of regional and seasonal products, local honeys and heritage grains, together with the integration of elements coming from the traditional wine production, such as the use of grape must and wine barrels [3]. Craft brewers reinterpret traditional beer styles varying the quantity and variety of hops and distinguishing their recipes using other raw or malted cereals (wheat, spelt, oat, rye, etc.) or adding fruit, spices, herbs, etc. As a result, they usually obtain a darker, stronger and more flavorful beer than the beers of mass producers [4].

As Italian beer consumers are very focused on quality and experimentation of different tastes, being very attentive on raw material and local origin [5], the interest on craft beer, brewed from local ingredients, is increasingly growing.

No studies have been carried out on consumers' preferences determining beer consumption [6] thus it is very important to explore beer perceptions using proper instruments. In recent years, a certain number of associations of "beer sommeliers" have flourished and often beer characterization relies on their personal experiences. However, this kind of beer characterization results in a nonobjective description less scientific than the sensory profile obtained by a trained panel of judges.

The present study reports different methods for describing craft beer sensory profile. A panel is trained for beer sensory analysis and five Tuscan craft beers are analyzed. Two different sensory analysis methods we reuse: a classic one, the Quantitative Descriptive Analysis, and Temporal Dominance of Sensations, a dynamic method. As consumer preferences and choices largely depend on the sensory properties and, in particular, on flavor perceptions [7], TDS is only focused on flavor attributes.

QDA method allows both the qualitative and quantitative description of beers producing the classic spider web sensory profiles. QDA is a complete sensory description, taking into account all sensations that are perceived (visual, tactile, olfactory, and gustatory) when the product is evaluated [8]. TDS, as a descriptive multi-attribute methodology, which deals with attribute interactions [9], allows the description of the overall product perception over time giving a real-time flavor profile [10]. TDS provides supplementary temporal information, perceived during the drinking process, on qualitative changes that are not measurable with the conventional sensory profile; moreover this method is less time consuming than other dynamic ones [7].

The scientific profiles obtained are compared with the description given by an expert beer taster in order to check for the correspondence between the trained panel perceptions and the expert description.

\section{Material and Methods}

\subsection{Sensory Analysis}

\subsubsection{Protocol for Sensory Analysis and Product Presentation}

A panel of 12 judges was selected and trained for beer sensory characterization. Commercial beers were used in pre-testing panel-test sessions to let the assessors familiarize with the products under investigation and the terminology related to it. Those sessions were also used to standardize panel's attributes definitions according to literature. All samples were presented in $80 \mathrm{ml}$ glass covered with a glass top and containing $20 \mathrm{ml}$ beer per glass for QDA and $10 \mathrm{ml}$ beer per glass for TDS. Samples were stored in fridge until prepared. Immediately before tasting they were poured and served at $10^{\circ} \mathrm{C}$. Samples were coded with a randomly selected three-digit number. Tests were carried out in individual booths and replicated twice. Water was supplied to rinse the palate between samples.

The experimental design was based on Latin squares to minimize the effect of serving order.

Data collection was performed with FIZZ software (Biosystemes, Couternon, France) through a computer network.

The five samples were craft beers manufactured in the area of Maremma, Tuscany, and were selected as representative regional products. Different aromatic features characterized the craft beers selected.

One Weiss high-fermentation beer, 6\% of alcohol, here reported as "W1" (Figure 1), one Weizen bock highfermentation beer, 6.5\% of alcohol, indicated as "W2" (Figure 2), one Pilsner low-fermentation beer, 4.5\% alcohol, indicated as "P1" (Figure 3), one Pilsner low-fermentation beer, 6.9\% alcohol, indicated as "P2" (Figure 4), and a red high-fermentation beer, 6.9\% alcohol, here reported as “R1” (Figure 5). 


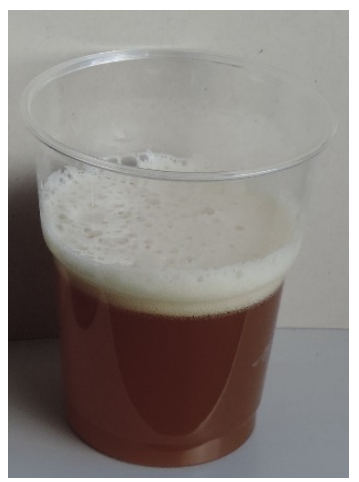

Figure 1. The Weiss beer W1.

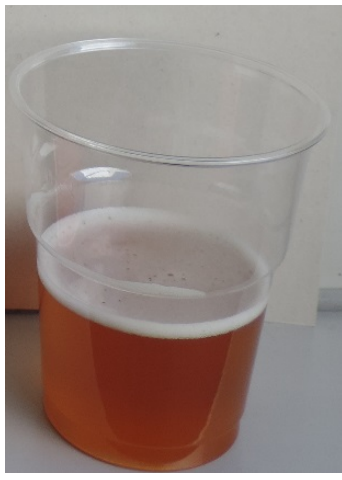

Figure 2. The Weizen bock beer W2.

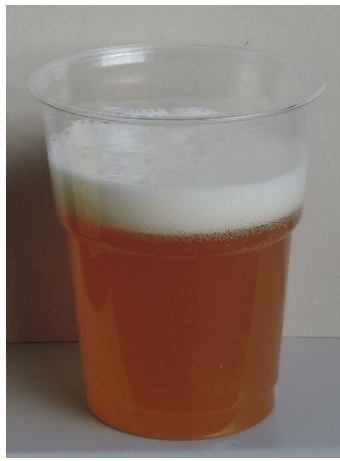

Figure 3. The Pilsner beer P1.

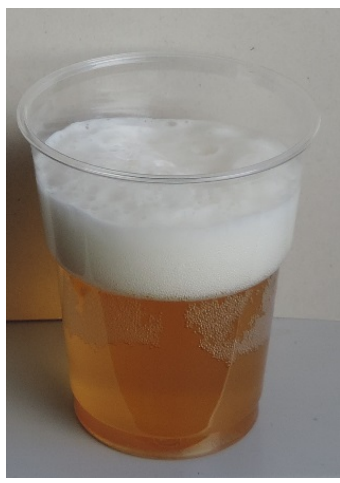

Figure 4. The Pilsner beer P2. 


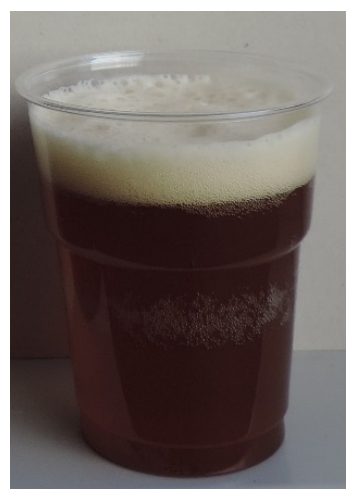

Figure 5. The red high-fermentation beer R1.

\subsubsection{QDA}

Twenty-eight attributes, (Table 1) derived from literature [11] and from the attribute list used by the "beer taster association" [12] were included in the evaluation process. Nine of them were related to odour (overall intensity, malty, hoppy, floral fruity, spicy, honey, roasted, other odours); two were visual attributes (foam persistency, turbidity), fourteen were gustatory traits (overall intensity, sweet, bitter, salty, sour, alcohol, malty, hoppy, floral fruity, spicy, honey, roasted, other flavors) and three of them concerned texture (fullness, level of carbonation, astringent).

The panelists were said to gradually sip the sample and describe it using the computer. The sensory attributes were assessed using an unstructured nine-point scale anchored at the left end with "absent" and at the right end with "high".

\subsubsection{TDS}

TDS analysis was focused on the aromatic phase and was performed using a subset of 7 aromatic attributes chosen among the descriptive ones used in QDA: floral, fruity hoppy, malty, honey, spicy and roasted. Panelists were trained to the TDS testing protocol through FIZZ Software (Biosystemes, Couternon, France). Each evaluation session lasted 90 seconds [10]. Panelists were asked to start the evaluation immediately after taking the sample $(10 \mathrm{ml})$ in their mouth. Panelists were then asked to check the dominant attribute perceived choosing it from the list. The dominant attribute was defined as: "the sensation catching the most the attention during the drinking process". An attribute remained dominant until replaced by another. Panelists were free to select the same attribute several times during the evaluation. The attribute list was randomized in order to minimize position errors. Panelists could stop the acquisition through a "stop" button when no more sensations were perceived, otherwise the evaluation automatically stopped after 90 seconds.

\subsubsection{Beer Description by the Expert}

An expert beer taster, a "beer sommelier" described the beers giving a characterization of each beer according to his own experience.

\subsubsection{Data Analysis}

Sensory data were processed using SAS 9.3 (SAS Institute Inc., Cary, NC, USA). Sensory profiles were defined by mean values. In TDS analysis, dominance rates (the percentage of panelists selecting a dominant attribute at a specific time) were calculated by attribute and time and were reported in TDS graphs through FIZZ Software (Biosystemes, Couternon, France).

Principal component analysis (PCA) was carried out on panel data to identify the key attributes mostly contributing to the variation in products within the product space. The beer codes on the PCA graph were then substituted to the short "beer sommelier" description in order to verify the correspondence.

\section{Results}

\subsection{QDA}

In descriptive analysis, W1 (Figure 6) showed high turbidity and quite low foam persistency. In the olfactory 
Table 1. Attribute list used in QDA analysis [4] [8].

\begin{tabular}{|c|c|}
\hline ODOUR ATTRIBUTE & \\
\hline OVERALL INTENSITY & Overall Odour intensity perceived \\
\hline MALTY & Aromatic note of fermented cereals similar to fresh malt cooked in the oven \\
\hline HOPPY & Typical odourof fresh hop \\
\hline FLORAL & Aromatic note resembling flowers \\
\hline FRUITY & Aromatic note resembling fruits \\
\hline SPICY & Aromatic note resembling spices \\
\hline HONEY & Aromatic sweet note resembling honey \\
\hline ROASTED & Aromatic note resembling caramel, bread crust, hazelnuts and browning of sugars \\
\hline \multicolumn{2}{|l|}{ VISUAL ATTRIBUTE } \\
\hline FOAM PERSISTENCY & Visual persistency of foam in the glass \\
\hline TURBIDITY & Presence of suspended particles that let the beer opaque \\
\hline \multicolumn{2}{|l|}{ GUSTATORY ATTRIBUTE } \\
\hline OVERALL INTENSITY & Overall Taste intensity perceived \\
\hline SWEET & The taste of sugar \\
\hline BITTER & The taste of substances like caffeine or quinine \\
\hline SALTY & Taste of salt \\
\hline SOUR & Taste of acidic substances such as citric acid \\
\hline ALCOHOL & Flavor sensation of alcohol \\
\hline MALTY & Flavor sensation of malt \\
\hline HOPPY & Flavor sensation of hop \\
\hline FLORAL & Flavor sensation resembling flowers \\
\hline FRUITY & Flavor sensation resembling fruits \\
\hline SPICY & Flavor sensation resembling spices \\
\hline HONEY & Flavor sensation resembling honey \\
\hline ROASTED & Flavor sensation resembling caramel, bread crust, hazelnuts and browning of sugars \\
\hline \multicolumn{2}{|l|}{ TEXTURE ATTRIBUTE } \\
\hline FULLNESS & Fullness of beer perceived in mouth \\
\hline LEVEL OF CARBONATION & Attribute resembling the pungency intensity of carbon dioxide \\
\hline ASTRINGENT & Mouth dry sensation \\
\hline
\end{tabular}

and gustative phase intensity, fruity, hoppy and spicy notes were highlighted. W2 (Figure 7), showed high foam persistency and turbidity and was mainly characterized by spicy and malty notes in the olfactory phase, while aromatic notes were characterized also by alcohol, sour, bitter and hoppy. P1 (Figure 8) had medium turbidity and foam persistency and was featured by hoppy, floral and fruity notes in the olfactory phase, while aromatic notes were mainly bitter alcohol and hoppy. P2 (Figure 9) had high foam persistency but low turbidity and was characterized by hoppy floral and honey olfactory notes that can be also found in the gustatory phase together with the attributes bitter and alcohol. R1 (Figure 10) had a medium foam persistency, while malty and roasted notes were highlighted in the olfactory phase and, bitter, roasted and alcohol notes in the gustatory phase. 


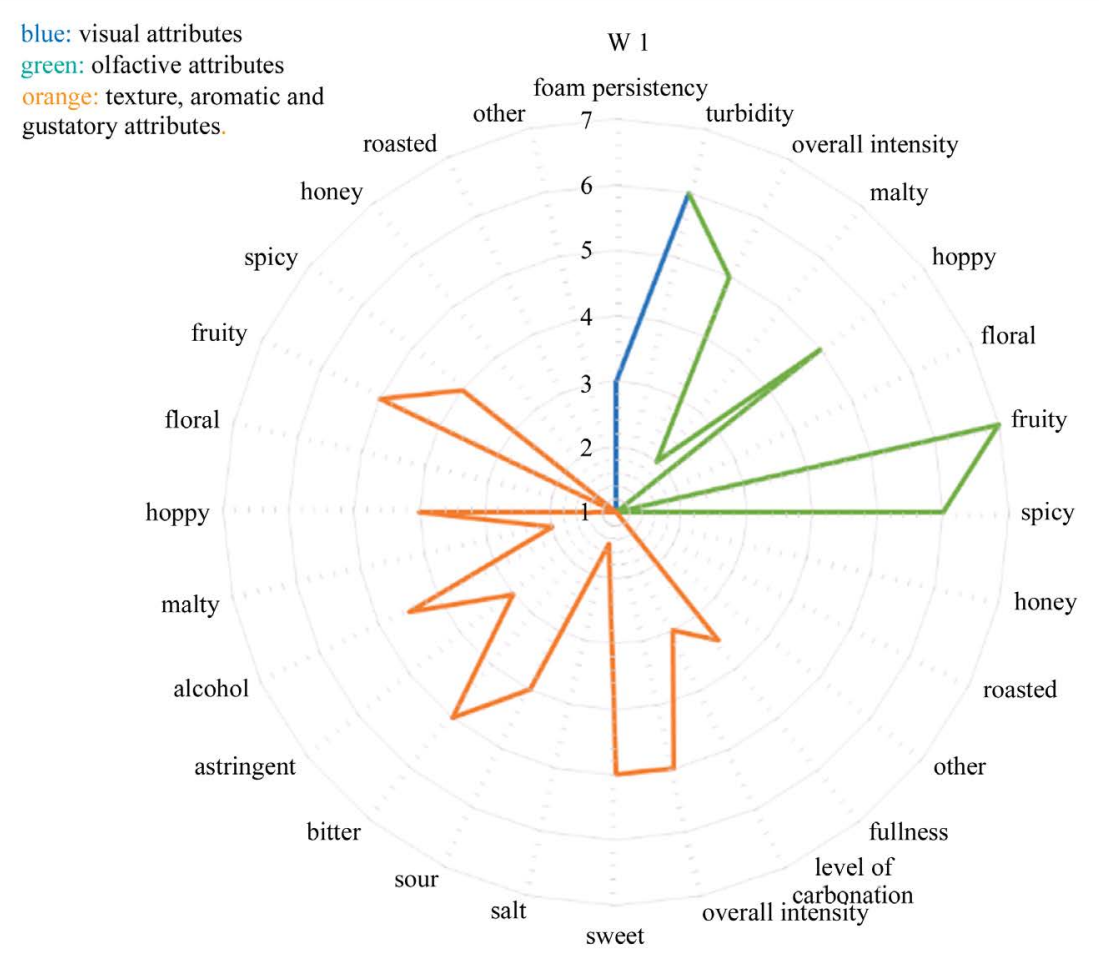

Figure 6. QDA of W1 beer.

blue: visual attributes

green: olfactive attributes

orange: texture, aromatic and

gustatory attributes.
W 2

other 7 turbidity

overall intensity

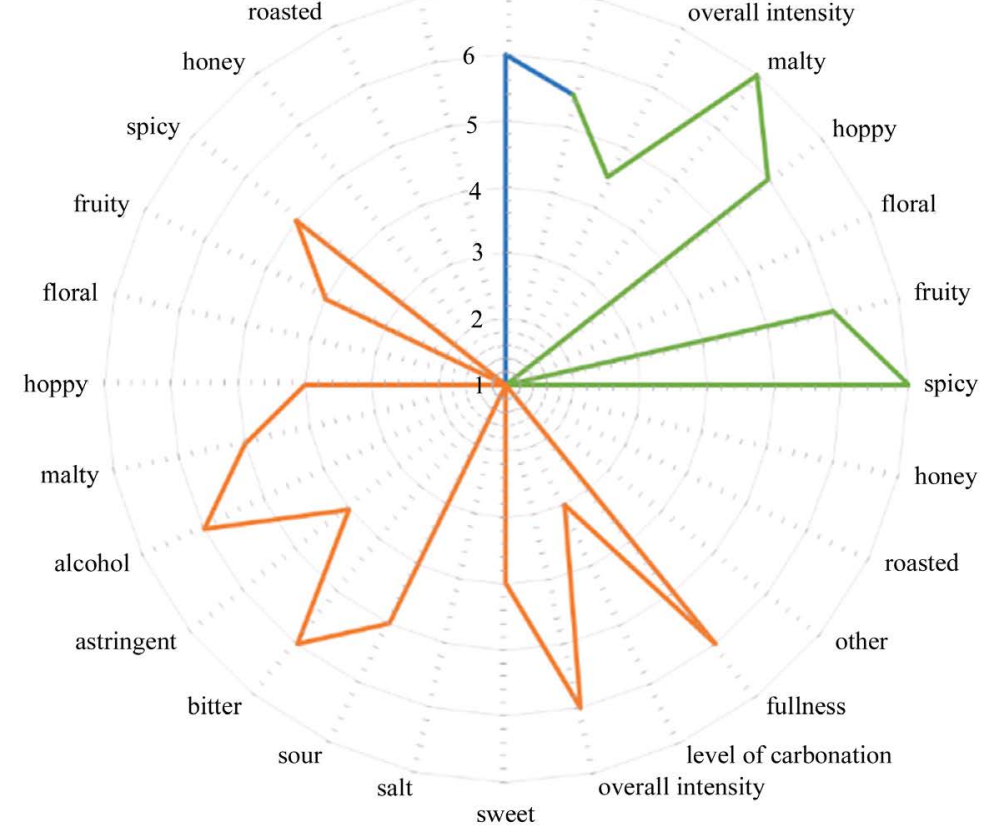

Figure 7. QDA of W2 beer. 
C. Medoro et al.

blue: visual attributes green: olfactive attributes orange: texture, aromatic and gustatory attributes
P 1

$$
\text { other } \begin{gathered}
\text { foam persistency } \\
7
\end{gathered} \text { turbidity }
$$

overall intensity

honey

6

spicy

5

malty hoppy

fruity 4 floral fruity spicy

floral

2

hoppy

malty

alcohol

astringent

bitter

sour

honey

roasted

other

fullness

level of carbonation

salt

sweet

Figure 8. QDA of P1 beer.

blue: visual attributes green: olfactive attributes orange: texture, aromatic and gustatory attributes
P 2

$$
\text { foam persistency }
$$

turbidity

overall intensity

7

roasted

other

honey

6

malty

spicy

fruity

floral

hoppy

5

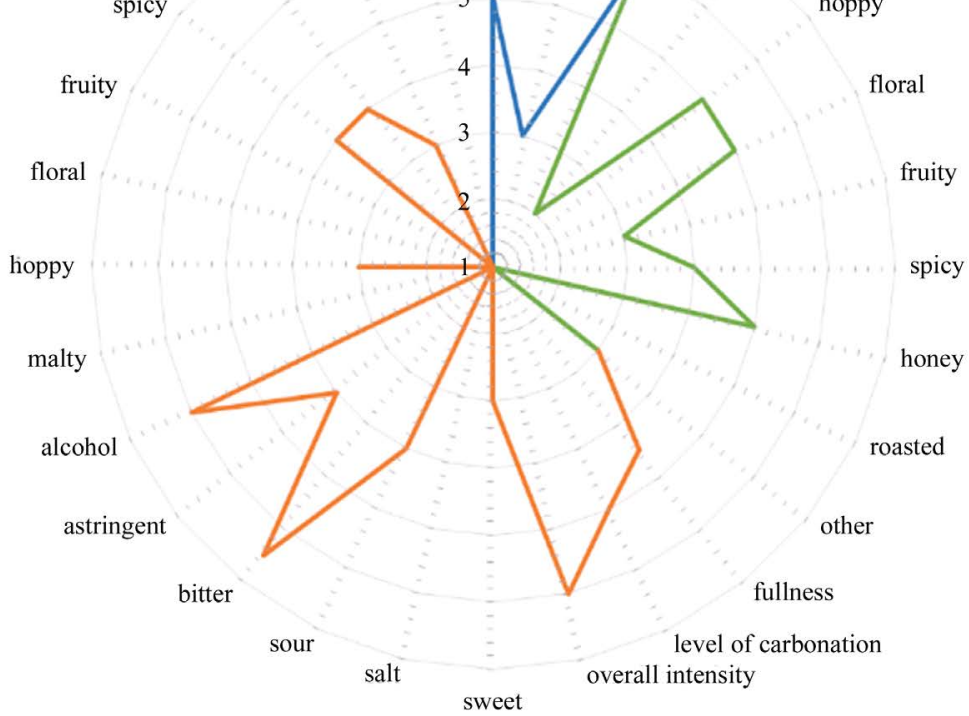

Figure 9. QDA of P2 beer.

460 


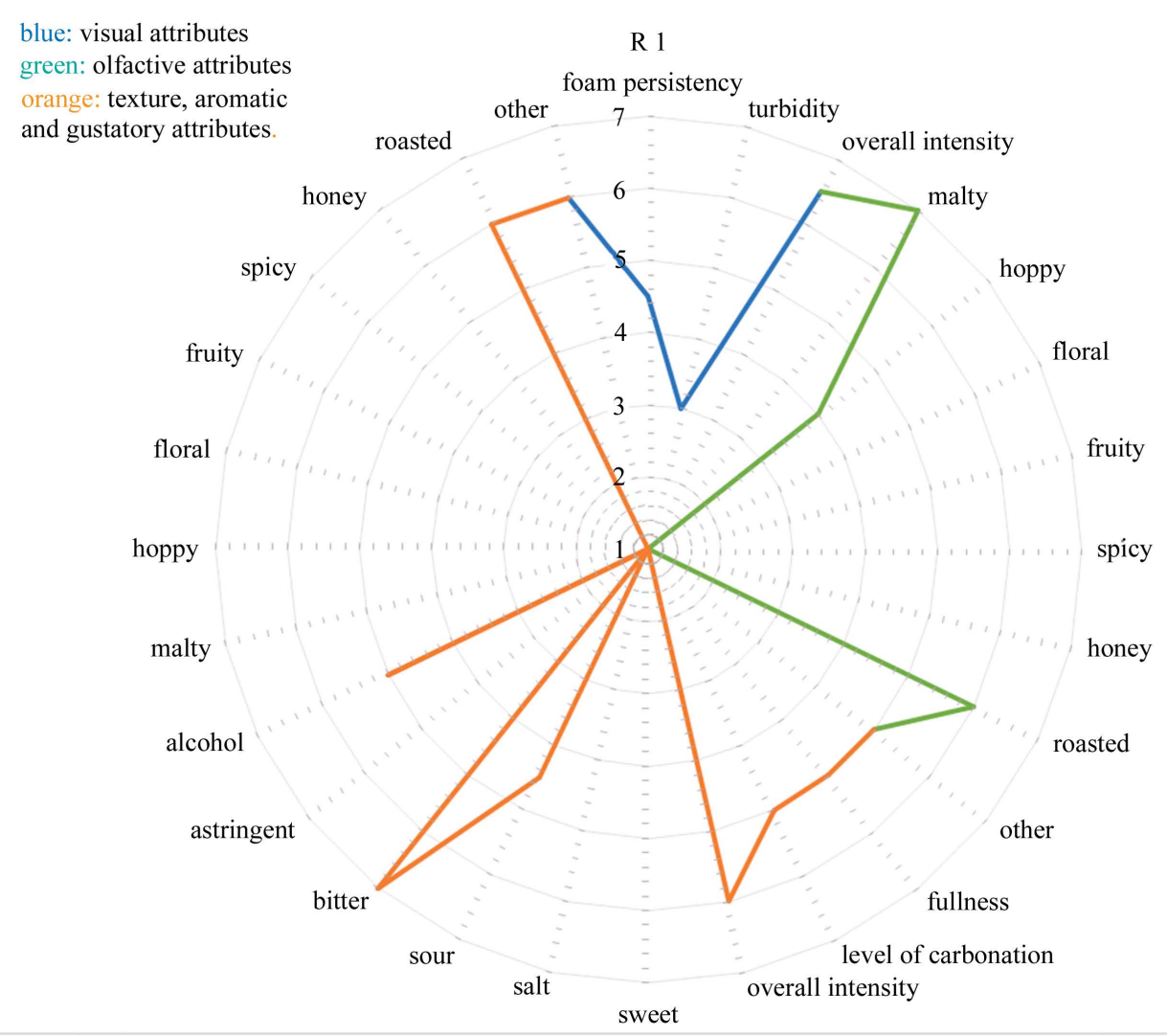

Figure 10. QDA of R1 beer.

\subsection{TDS}

TDS aromatic curves showed, in W1 (Figure 11), the dominance and persistence of fruity notes over all the attributes, but also malty and spicy notes were indicated as dominant. W2 (Figure 12) showed a more complex scenario in which different balanced aromatic notes arose: spicy, malty, fruity and hoppy were the most dominant sensations. P1 (Figure 13) was characterized by the dominance of hoppy, malty and roasted. Spicy was also present by the end of the tasting period. P2 (Figure 14) was dominated by honey, fruity and hoppy notes during all of the tasting period. R1 (Figure 15) was mainly dominated by roasted notes with the other attributes only slightly dominant during the tasting period.

\subsection{Beer Descriptions by the Expert}

The "beer sommelier" after tasting the five craft beers gave the following descriptions. W1: a fruity balanced and harmonic "Weiss" characterized by sweet notes of "banana", spices and hop. W2: a "Weizen bock" characterized by alcoholic, spicy, malty and sour notes. A "young" but well balanced and harmonious beer. P1: a mature "pilsner" with bitter and floral notes. The P1 complexity of taste notes was quite low but it was a rather balanced and harmonious beer. P2: a strong "pilsner" characterized by bitter, honey, hoppy and spicy notes. The P2 taste was harmonious but mildly balanced. R1: a full-bodied red beer, with roasted, malty and bitter notes. R1 was featured by a rather balanced and harmonious taste.

\subsection{Comparison of QDA Profiles to the Expert Beer Taster Descriptions}

The PCA (Figure 16) showed the most contributing attributes to beer profiles. The first two principal components of PCA accounted for $74 \%$ of the variance in the data. The descriptions given by the beer taster expert were in agreement with the attributes identifying the beers in QDA profiles as showed in Figure 11. PC1 and PC2 differentiated the two "Pilsner", P1, P2, mainly floral and bitter, from the two "Weiss" beers, W1 and W2, 
W1

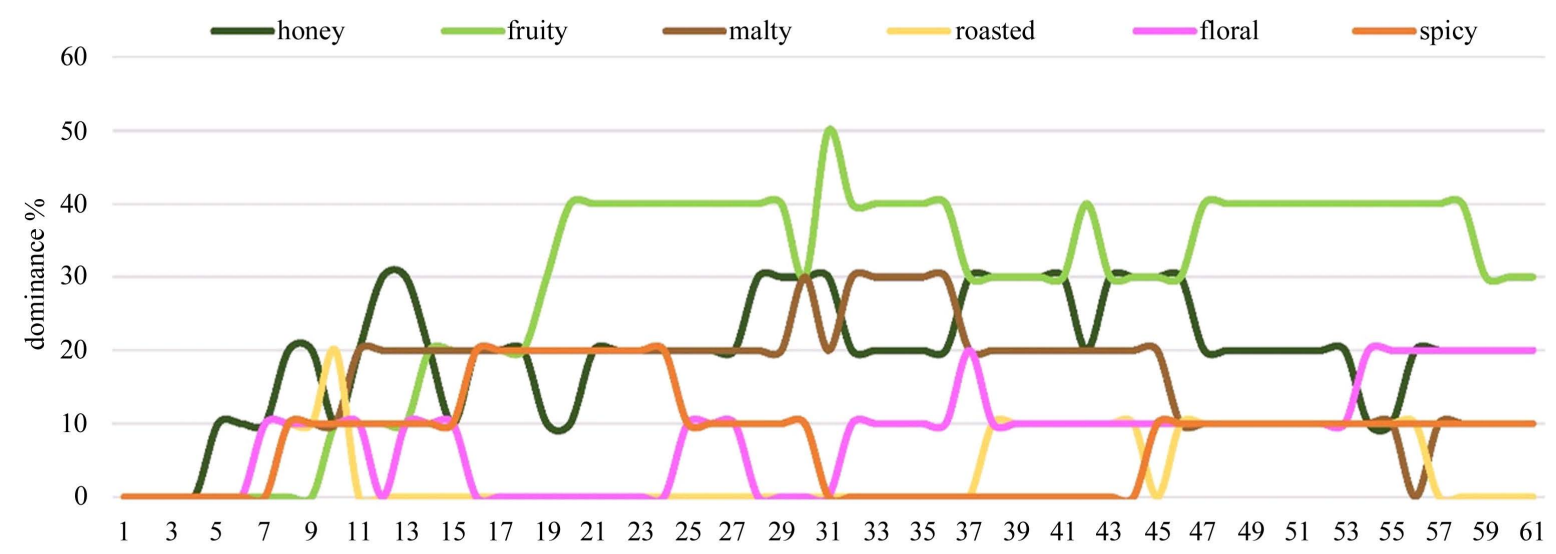

$\mathrm{T}$ (s)

Figure 11. TDS of W1 beer.

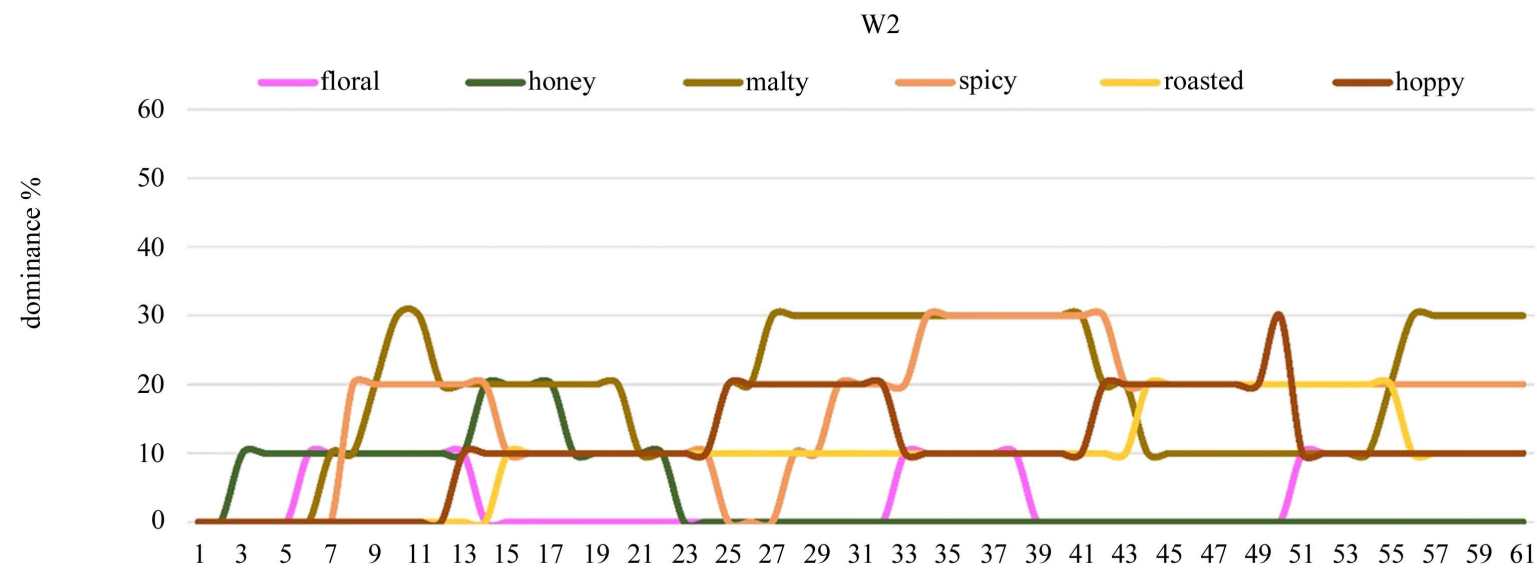

$\mathrm{T}$ (s)

Figure 12. TDS of W2 beer.

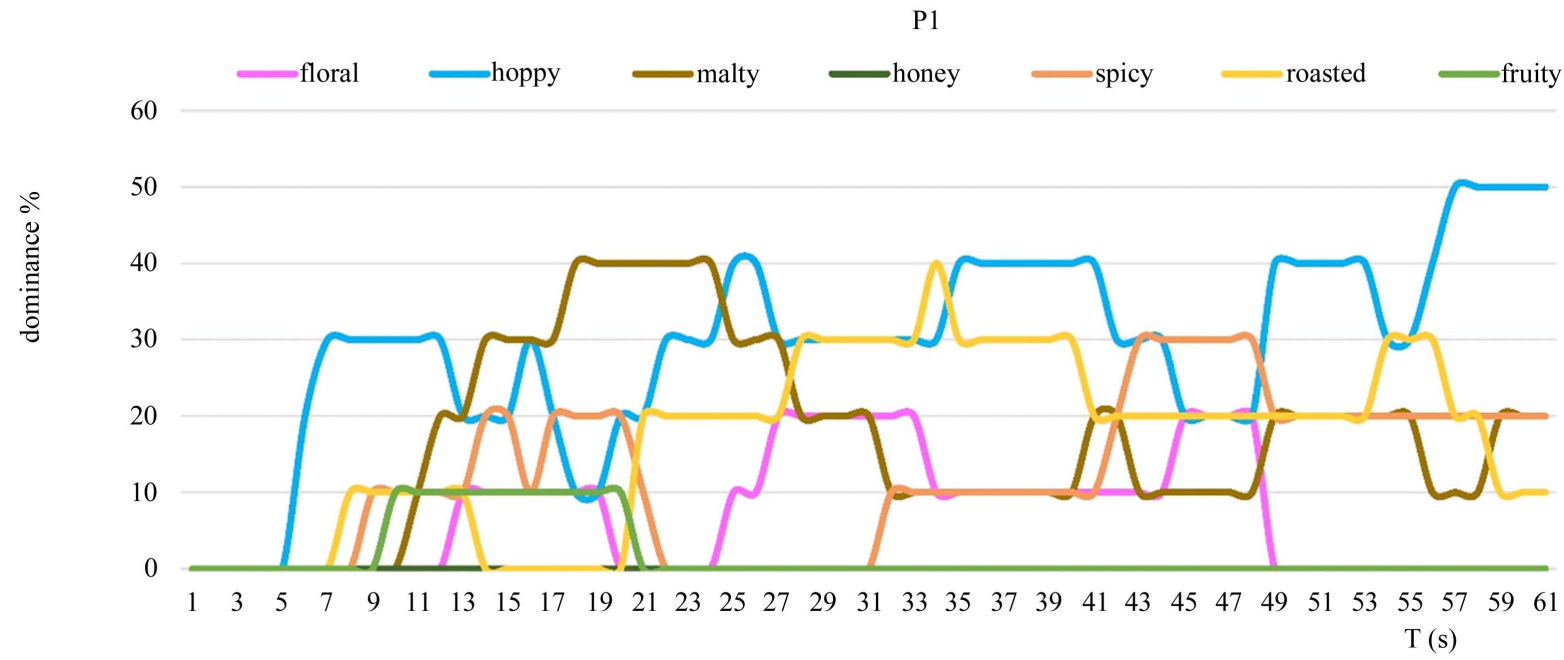

Figure 13. TDS of P1 beer. 


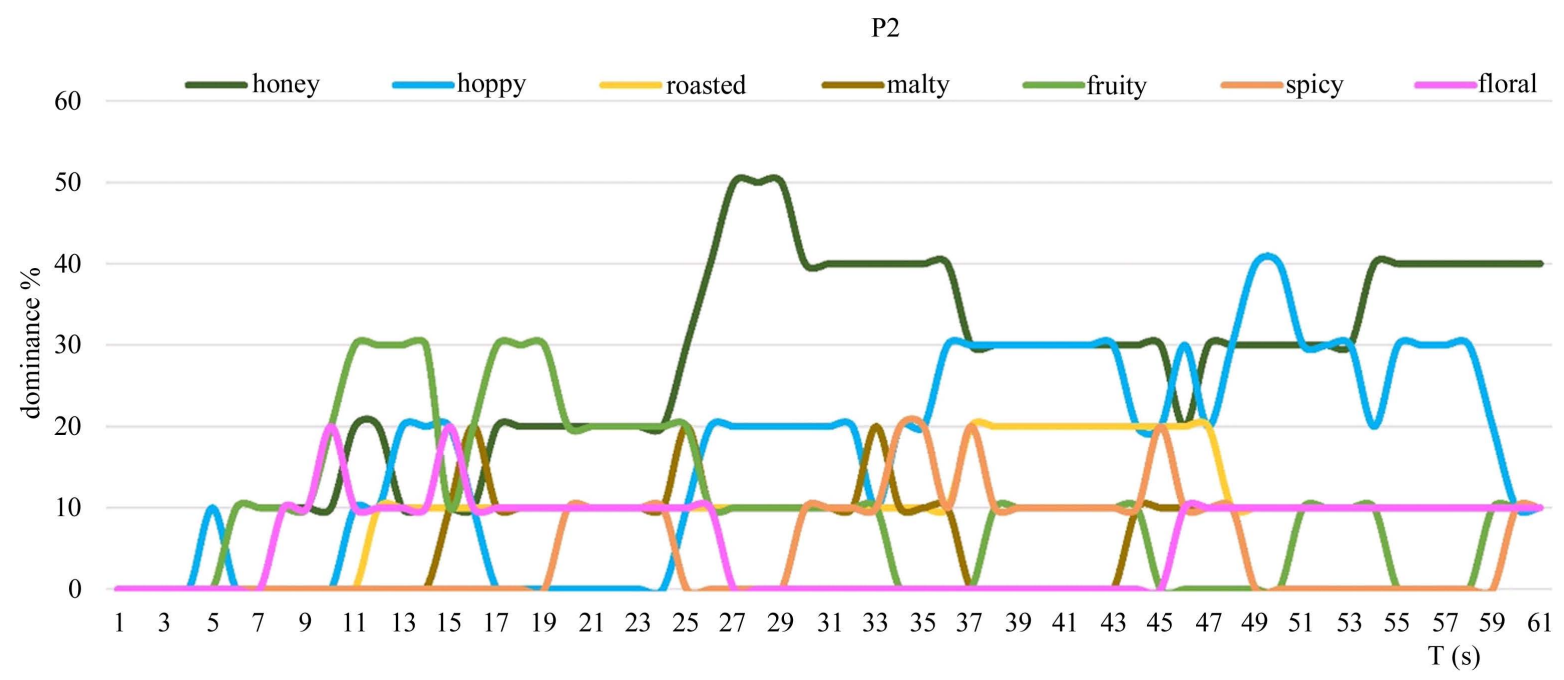

Figure 14. TDS of P2 beer.

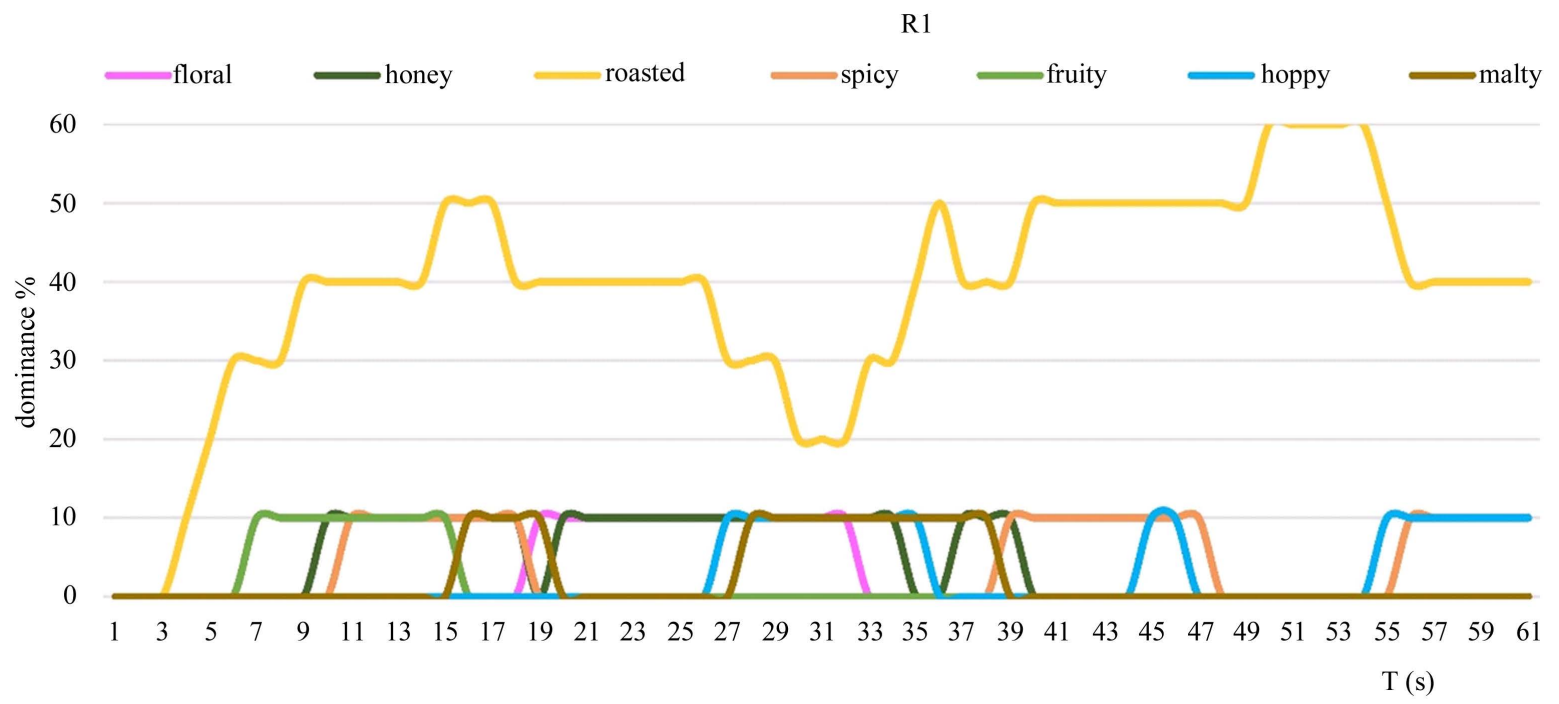

Figure 15. TDS of R1 beer.

featured by fruity and spicy notes, R1 was not close to any of the other beers mainly featured by roasted and malty notes.

\section{Discussion}

Recently, Italian beer industry has been showing a growing trend [3], but according to AssoBirra, the Italian Association of Brewers, also the interest on craft beer manufacturing and consumption has been rising [13] [14]. The main traits of craft brewers, the interest and the exploration on flavors and brewing techniques [15], lead to the formulation of products characterized by complex pools of flavors, high quality and great appeal for consumers [3]. Therefore, craft beers need proper analytical instruments to be described and explored. In this work, the complexity of flavors of five Italian craft beers, manufactured in Maremma (Tuscany), were described through classic and dynamic profile methods. The results of this study proved that these methods are comparable to the description given by the expert taster.

The results of the TDS analysis applied on the five craft beers have shown to be mostly in agreement with those obtained by the QDA. The outcomes of both the analytical methodologies emphasized the flavor aspects of the craft beers, revealing the complexity of craft beers flavors and the dynamic of their modification during 


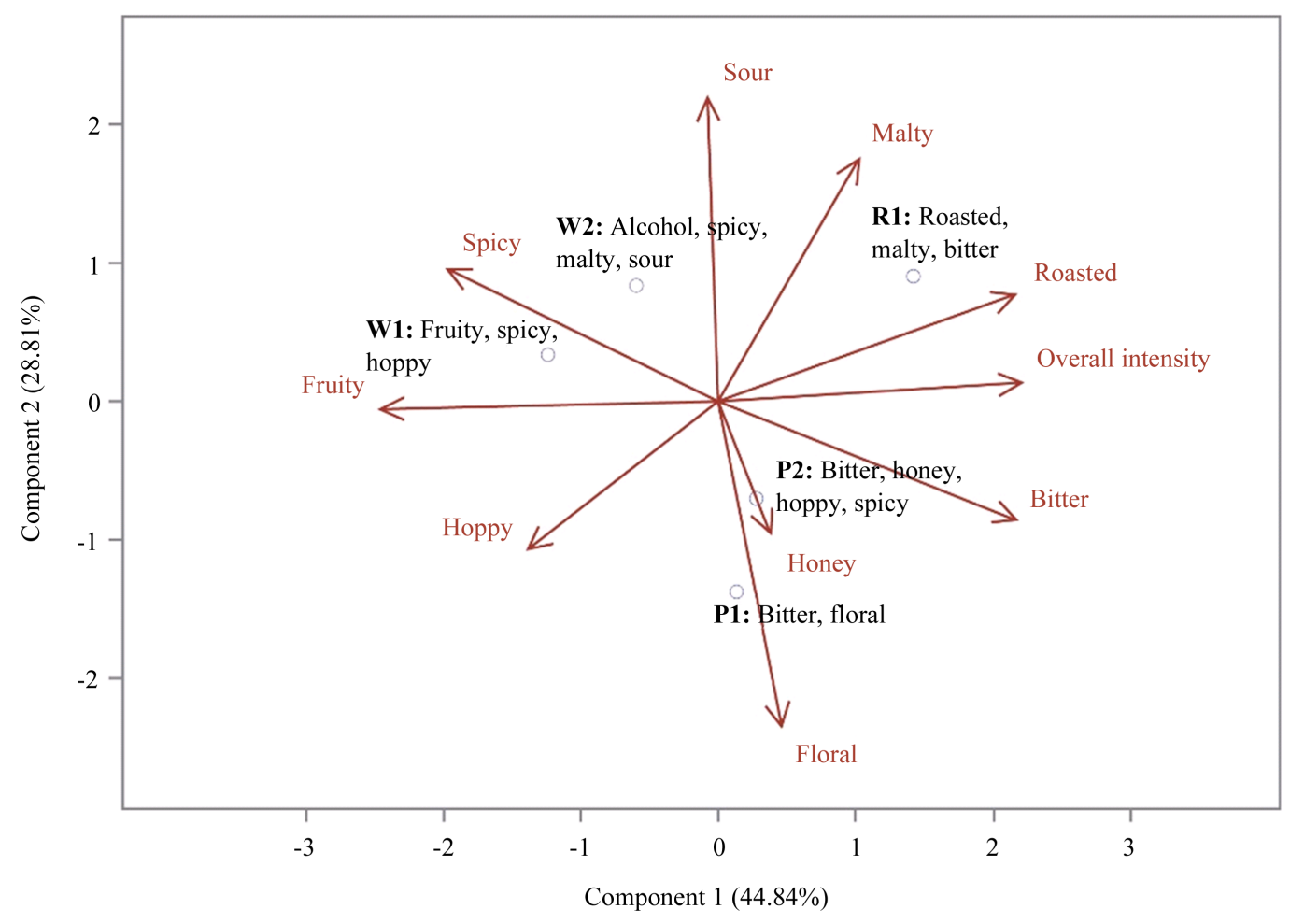

Figure 16. PCA analysis of the five Tuscan beers. Attributes resuming beer features are in red. Codes of the five beers are in bold letters followed by a summary of the "sommelier" description.

tasting. Furthermore, accordance was found between the sommelier descriptions of the five beers and the sensory data obtained by the trained panel, as showed by PCA analysis. In such a way, the results validate the panel activity of the twelve judges that corresponds to the description given by the beer "sommelier", an expert who is officially acknowledged by the Italian national organization of beer tasters and to whom traditionally breweries referred to for their description. Moreover, results of this study showed that the two sensory analytical methods were effective and suitable instruments to study organoleptic properties and exhaustively achieve the sensory profiles of craft beers.

\section{Conclusion}

The results of this work are interesting and useful information for brewers and craft brewers that can take the chance of choosing different methods to describe sensory features of their brewery products. Sensory analysis methods can be a good and more scientific alternative to the "sommelier" classic beer description. Sensory profiles, obtained through different available sensory techniques, can be a fundamental support for entrepreneurs in targeting specific segments of the beer market. By applying sensory analysis in defining the organoleptic attributes, craft beer can get added value and be more recognizable and properly marketable in particular sectors, included the rural tourism one. Actually sensory analysis has shown to be an effective tool for educating consumers/tourists in achieving critical understanding and awareness of the product as well as recognizing the uniqueness of organoleptic cues and designing memorable experiences. So, sensory analysis can emphasize the craft market products, such as artisanal beers, and can be conceived as a catalyst for enhancing the effects of a differentiation strategy in business [16].

\section{Acknowledgements}

We would like to thank the form Province of Grosseto, Dept. of Rural Development for financing this research and, particularly, Mr. Valter Nunziatini and Ms Violetta Zamperini for supporting the organization of the work. We would also like to acknowledge local craft breweries for providing beer products. 


\section{References}

[1] Barth-Haas Group (2006) The Barth Report: Hops 2005/2006. Germain Hansmaennel, Strasbourg.

[2] Reinbach, H.C., Giacalone, D., Machado Ribeiro, L., Bredie, W.L.P. and Bom Frøst, M. (2014) Comparison of Three Sensory Profiling Methods Based on Consumer Perception: CATA, CATA with Intensity and Napping. Food Quality and Preference, 32, 160-166. http://dx.doi.org/10.1016/j.foodqual.2013.02.004

[3] Biancolillo, A., Bucci, R., Magrì, A.L., Magrì, A.D. and Marini, F. (2014) Data-Fusion for Multiplatform Characterization of an Italian Craft Beer Aimed at Its Authentication. Analytica Chimica Acta, 820, 23-31. http://dx.doi.org/10.1016/j.aca.2014.02.024

[4] Fastigi, M., Espost, R., Orazi, F. and Vigano, E. (2015) The Irresistible Rise of the Craft Brewing Sector in Italy: Can We Explain It? 4th AIEAA Conference-Innovation, Productivity and Growth, Ancona, 11-12 June 2015, 1-23.

[5] Mascia, I., Fadda, C., Dostàlek, P., Olsovkà, J. and Del Caro, A. (2014) Preliminary Characterization of an Italian Durum Wheat Beer. Journal of the Institute of Brewing, 120, 495-499.

[6] Aquilani, B., Laureti, T., Poponi, S. and Secondi, L. (2014) Beer Choice and Consumption Determinants When Craft Beers Are Tasted: An Exploratory Study of Consumer Preferences. Food Quality and Preference, 41, 214-224. http://dx.doi.org/10.1016/j.foodqual.2014.12.005

[7] Déléris, I., Saint-Eve, A., Guo, Y., Lieben, P., Cypriani, M.-L., Jacquet, N., Brunerie, P. and Souchon, I. (2011) Impact of Swallowing on the Dynamics of Aroma Release and Perception during the Consumption of Alcoholic Beverages. Chemical Senses, 36, 701-713. http://dx.doi.org/10.1093/chemse/bjr038

[8] Stone, H., Bleibaum, R.N. and Thomas, H.A. (2012) Sensory Evaluation Practices: Descriptive Analysis. Elsevier, USA, 233-289.

[9] Pineau, N., Schlich, P., Cordelle, S., Mathonniere, C., Issanchou, S., Imbert, A., Rogeaux, M., Etievant, P. and Koster, E. (2009) Temporal Dominance of Sensations: Construction of the TDS Curves and Comparison with Time-Intensity. Food Quality and Preference, 20, 450-455. http://dx.doi.org/10.1016/j.foodqual.2009.04.005

[10] Vazquez-Araujo, L., Parker, D. and Woods, E. (2013) Comparison of Temporal-Sensory Methods for Beer Flavor Evaluation. Journal of Sensory Studies, 28, 387-395. http://dx.doi.org/10.1111/joss.12064

[11] Donadini, G., Fumi, M.D. and Newby-Clark, I.R. (2014) Consumers’ Preference and Sensory Profile of Bottom Fermented Red Beers of the Italian Market. Food Research International, 58, 69-80. http://dx.doi.org/10.1016/j.foodres.2014.01.048

[12] Associazione Degustatori di Birra. http://www.degustatoribirra.it

[13] AssoBirra (2012) Annual Report 2011. 1-99. http://www.assobirra.it/press/wp-content/ar2012_assobirra1.pdf

[14] AssoBirra (2013) Annual report 2012. http://www.assobirra.it/press/wp-content/ar-assobirra-2012-ok.pdf

[15] Oliver, G. (2011) Craft Brewing. The Oxford Companion to Beer, Oxford University Press, Oxford, 270-271.

[16] Santini, C., Cavicchi, A. and Canavari, M. (2011) The Risk ${ }^{\mathrm{TM}}$ Strategic Game of Rural Tourism: How Sensory Analysis Can Help in Achieving a Sustainable Competitive Advantage. Springer, Heidelberg Dordrecht, London, New York, 161-179. 